\title{
Employing an Enhanced Interval Approach to Encode Words into Linear General Type-2 Fuzzy Sets for Computing With Words Applications
}

\author{
Aysenur Bilgin, Hani Hagras \\ School of Computer Science and Electronic Engineering \\ University of Essex \\ Colchester, UK \\ abilgin@essex.ac.uk
}

\author{
Daniyal Alghazzawi, Areej Malibari, Mohammed J. \\ Alhaddad \\ Faculty of Computing and Information Technology \\ King Abdulaziz University \\ Jeddah, Saudi Arabia
}

\begin{abstract}
In 1996, Zadeh coined Computing With Words (CWWs) to be a methodology in which words are used instead of numbers for computing and reasoning. One of the main challenges which faced the CWWs paradigm has been modelling words adequately. Mendel has pointed out that the CWWs paradigm should employ type-2 fuzzy logic to model words. This paper proposes employing an Enhanced Interval Approach (EIA) to create Linear General Type-2 (LGT2) fuzzy sets from Interval Type-2 (IT2) fuzzy sets to encode words for CWWs applications. We have performed experiments on 18 words belonging to 3 different linguistic variables (having 6 linguistic terms each). Interval data has been collected from 17 subjects and 18 linguistic terms have been modeled with IT2 fuzzy sets using EIA. The proposed conversion approach uses several key points within the parameters of IT2 fuzzy sets to redesign the linguistic variable using LGT2 fuzzy sets. Both IT2 and LGT2 fuzzy sets have been evaluated within a CWWs Framework, which aims to mimic the ability of humans to communicate and manipulate perceptions via words. The comparison results show that LGT2 fuzzy sets can be better than IT2 fuzzy sets in mimicking human reasoning as well as learning and adaptation since the progressive Root Mean Squared Error (RMSE) and Mean Absolute Percentage Error (MAPE) values for LGT2 based CWWs Framework converge faster and are lower than those for IT2 based CWWs Framework.
\end{abstract}

Keywords-linear general type-2 fuzzy sets, interval type-2 fuzzy sets, computing with words, enhanced interval approach

\section{INTRODUCTION}

At the early 70s, Zadeh [1] defined the concept of a linguistic variable which is one of the main foundations for the Computing With Words (CWWs) paradigm. In 1996, the CWWs paradigm was explicitly put forward by Zadeh [2] to be a methodology in which words are used instead of numbers for computing and reasoning. Later on, Zadeh elaborated on the purpose of CWWs which was defined to mimic the ability of humans to communicate and manipulate perceptions via words [3]. Accordingly, since words have fuzzy denotations as used by humans, there is a need to deal with imprecision; and fuzzy logic is a methodology that serves this purpose [2].

After having established the linkage between CWWs and fuzzy logic in general, it was pointed by Mendel [4] that the CWWs paradigm is associated with type-2 Fuzzy Logic (FL). According to Mendel [4] 'words mean different things to different people' and the linguistic uncertainties that are inherent in the 'words' require type-2 FL as type-1 FL cannot handle such uncertainties. In his work [5], Mendel states that using a type-1 fuzzy set to model a word is scientifically incorrect, because a word is uncertain whereas a type-1 fuzzy set is certain. Furthermore, in [4], the Footprint of Uncertainty (FOU) (which is involved in type-2 fuzzy logic) for a word was obtained from people via surveys and the linguistic uncertainty was represented within the interval information. By using this interval information in the construction of type-2 fuzzy sets, Mendel [4] demonstrates that the uncertainty has the potential to reduce complexity as the number of type- 2 membership functions for representing the labels were found to be less than that of type-1 membership functions where the uncertainty is ignored. In essence, Mendel encourages the use of type-2 FL (in particular interval type-2 (IT2) FL [6], [7], [8]) to adequately encode words for Per-C, which is an architecture for CWWs paradigm. Liu and Mendel proposed an Interval Approach (IA) in [9] to synthesize an IT2 fuzzy set model for a word, in which, interval endpoint data about a word are collected from a group of subjects where the subjects are asked: On a scale of $0-10$, what are the endpoints of an interval that you associate with the word ... ?. In this approach, each subject's data interval is mapped into a type-1 fuzzy set; and an IT2 fuzzy set model is obtained for the word from several type-1 fuzzy sets. However, there were some limitations to the IA approach, which were addressed by the Enhanced Interval Approach (EIA) proposed by $\mathrm{Wu}$, Mendel and Coupland [10].

In our previous work [11], the building block of CWWs paradigm, which is a word, has been broken down to parts of speech in English language in order to design better representations of words that are essential in human communication. We have argued that not all types of fuzzy sets are sufficient to represent words for CWWs paradigm. In addition to the inadequacies of type-1 fuzzy sets, which were already mentioned by Mendel, we have recapitulated the shortcomings of IT2 fuzzy sets, which are prone to modeling a number of incompatible statements as pointed in [12]. Consequently, to address these limitations, we have introduced a novel type of fuzzy set named Linear General Type-2 (LGT2) fuzzy set, which is inspired from linear adjectives [13], antonyms [14] and modifiers [15]. 
In this paper, we will present a conversion paradigm that converts IT2 fuzzy sets, which are created using EIA, into LGT2 fuzzy sets. We will show comparison analysis of IT2 fuzzy sets and LGT2 fuzzy sets that are deployed in a CWWs framework, which has been previously introduced in [16], [17]. In compliance with the objective of CWWs paradigm, which is to mimic human reasoning through words, we will show that LGT2 fuzzy sets are better able to capture and hence model perceptions for CWWs paradigm.

The rest of the paper is organized as follows: Section II will briefly describe the shortcomings of type-1 and IT2 fuzzy sets from several perspectives including fuzzy logic and linguistics. Section III will formally introduce the mathematical definition of LGT2 fuzzy sets to overcome the inconsistencies of type-1 and IT2 fuzzy sets. In Section IV, we will present the proposed conversion paradigm which takes several key points obtained from the parameters of IT2 fuzzy sets created using EIA and maps these into the parameters of LGT2 fuzzy sets to redesign the word models. Section V, will present an overview on the deployed CWWs architecture that has been used in the realworld experiments. Section VI will present the experiments and results. Finally, we will draw conclusions in Section VII.

\section{SHORTCOMINGS OF TYPE-1 AND INTERVAL TYPE-2 FuZZY SETS IN MODELLING WORDS FOR CWWS PARADIGM}

In this section, we will briefly recapitulate the inconsistencies inherent to type-1 and IT2 fuzzy sets in modelling words for CWWs paradigm.

\section{A. Fuzzy Semantics of Type-1 Fuzzy Sets}

In addition to Mendel's [5] claim for the scientific inadequacy of type-1 fuzzy sets in modelling words, Klein [13]had also noted that natural ordering on real numbers can be lost in (type-1) fuzzy semantics. This condition in his work is described as follows [13]: For all $u, u^{\prime} \in U, \mu_{\text {tall }}(u)=$ $\mu_{\text {tall }}\left(u^{\prime}\right)$, if $u$ is exactly as tall as $u^{\prime}$ where tall is a fuzzy set, $\mu_{\text {tall }}(u)$ is the degree of membership in tall, and $U$ is the universe of discourse. To interpret the claim "Bill is taller than Tom is" in fuzzy semantics using the information $\mu_{\text {tall }}($ Bill) and $\mu_{\text {tall }}($ Tom $)$, one would obviously let $\mu_{\text {tall }}($ Bill $)>$ $\mu_{\text {tall }}($ Tom $)$, where $>$ is the natural ordering on the real numbers[13]. However, this conflicts with the reasonable assumption that if an individual $u$ reaches a certain height, say six foot three, then $u$ is definitely tall and hence $\mu_{\text {tall }}(u)=1$ (the case of shoulder membership functions). Hence, if Bill is six foot four, while Tom is six foot three, $\mu_{\text {tall }}($ Bill $)=$ $\mu_{\text {tall }}($ Tom), the claim "Bill is taller than Tom is" comes out false[13].

\section{B. Inconsistency of Interval Type-2 Fuzzy Sets}

Greenfield and John [12] show how an interval type-2 fuzzy set is capable of modelling a number of incompatible statements. In [12], the propositions under different types of logic are discussed using the statement $S=$ \{the perpetrator is tall. . Accordingly, in crisp logic, the statement $S$ is equivalent to the following [12]:
$\mathrm{S}_{\text {crisp }}=\{$ 'The perpetrator is tall.' is true. $\}$.

On the other hand, in type-1 fuzzy logic, the statement S can take the form of [12]: 0.8.\},

$S_{\text {type-1 }}=\{$ 'The perpetrator is tall.' has a truth value of

whereas in IT2 fuzzy logic, the statement $S$ can take the following forms [12]:

$\mathrm{S}_{\mathrm{IT} 2}=\{$ The statement $\{$ 'The perpetrator is tall.' has a truth value of 0.8$\}$ has a truth value of 1.$\}$.

$\mathrm{S}_{\mathrm{IT} 2}{ }^{\prime}=\{$ The statement $\{$ 'The perpetrator is tall.' has a truth value of 0.5$\}$ has a truth value of 1.$\}$.

Hence, it has been demonstrated that the statements $S_{\text {IT2 }}$ and $\mathrm{S}_{\mathrm{IT2}}{ }^{\prime}$ are inconsistent [12]. To address this, Greenfield and John propose the use of general type- 2 fuzzy logic to make the statements $\mathrm{S}_{\mathrm{GT} 2}$ and $\mathrm{S}_{\mathrm{GT} 2}{ }^{\prime}$ consistent as follows [12]:

$\mathrm{S}_{\mathrm{GT} 2}=$ \{ The statement $\{$ 'The perpetrator is tall.' has a truth value of 0.8$\}$ has a truth value of 1.$\}$.

$\mathrm{S}_{\mathrm{GT2}}{ }^{\prime}=\{$ The statement $\{$ 'The perpetrator is tall.' has a truth value of 0.5$\}$ has a truth value of 0.6 . $\}$.

In order to address the abovementioned drawbacks of type1 and IT2 fuzzy sets, which might cause inadequate representation of words for a real-world CWWs paradigm, a general type-2 fuzzy logic based approach focusing on human interpretability of words has been developed. The next section will formally introduce the linguistically inspired Linear General Type-2 Fuzzy Sets.

\section{LINEAR GENERAL TYPE-2 FUZZY SETS}

In this section, we will present a special kind of general type2 fuzzy set termed Linear General Type-2 Fuzzy Sets (LGT2 FSs) where the third dimension is quantified in a linear fashion. The theoretical formulation of LGT2 FSs is based on linear adjectives [13], antonyms [14] and modifiers [15]. From the linguistics perspective, we observed that the words (i.e. linguistic terms for linguistic variables) used in fuzzy logic are possibly adjectives (e.g. hot, cold, high, low, etc.), which have the distinctive characteristic of gradability [13] as they are modelled in a sortal range ${ }^{1}$ within their mathematical domain. Formally, given that $A$ is an adjective, Klein [13] puts forward two types of adjectives classified according to the following condition: "Whenever $c$ is a context of use, $N P_{1}, N P_{2}$ denote individuals within the sortal range of $A$, then the sentence $N P_{1}$ is $A-$ er than $N P_{2}$ has a definite truth value in $c$. ." [13]. Accordingly, the linear adjectives are those that satisfy this condition and the ones that do not are called to be nonlinear [13]. For example, let $c$ be a context of temperature, $N P_{1}=43$ and $N P_{2}=40$ within the sortal range of $={ }^{\prime} h o t^{\prime}$, then the sentence " 43 is hot $(t)$ - er than 40 " has a definite truth value in temperature context; therefore, 'hot' is a linear adjective as it satisfies the above condition.

From another linguistics perspective, Kennedy [15] presents a decompositional approach where he argues that "... the 
meaning of a gradable adjective contains a measure function" [15]. To illustrate, let ' $h o t$ ' be a gradable adjective and 'extremely' be a measure function which determines the degree to which a variable $\mathrm{x}$ is 'hot'; in this case 'extremely' alone is not the core meaning of the adjective according to [15]. In linguistics literature, modifiers as measure phrases have been studied in detail [18]-[20] where it is agreed that semantics of measure phrases require an adjustment in the meaning of an adjective [19]. However, the adjustments caused by measure phrases (i.e. modifiers) also introduce linguistic uncertainties.

Thus, in order to model a word for CWWs, there is a need to deal with the linguistic uncertainty that modifiers encapsulate as their level of intensifying or diminishing the meaning of an adjective changes from one person to another. For example, when the modifier 'extremely' is used to intensify the meaning of an adjective, it might mean different amount of intensifications to different people. With the aim to handle the linguistic uncertainty conveyed by modifiers in a novel way, we model the modifiers as second-order word uncertainty. The point of departure for this is twofold: 1) there exists a hierarchical analogy between the linear adjectives and a linguistic variable in a fuzzy system [11] 2) as mentioned by [15], the major meaning of the linguistic label is delivered by the adjective and this semantically justifies modelling the adjective as first-order uncertainty.

\section{A. Mathematical Definition of LGT2 Fuzzy Sets}

Formally, a type-2 FS, denoted $\tilde{A}$, can be expressed as follows [21]:

$$
\tilde{A}=\int_{x \in X} \int_{u \in J_{x}} \mu_{\tilde{A}}(x, u) /(x, u) \quad J_{x} \subseteq[0,1]
$$

Likewise, a Linear General Type-2 FS denoted L(Fig. 1a) can be expressed as follows:

$$
\tilde{L}=\int_{x \in X} \int_{u \in J_{x}} \mu_{\tilde{L}}(x, u) /(x, u) \quad J_{x} \subseteq[0,1]
$$

As shown in Fig. 1b, a vertical slice of $\mu_{\tilde{L}}(x, u)$ at $x=x^{\prime}$ can be formalized as follows: Let the primary membership of $\tilde{L}$ be represented by a type-1 shoulder Upper Membership Function (UMF) whose parameters are denoted as $\left[a_{u}, b_{u}, c_{u}, d_{u}\right]$ and a type-1 Lower Membership Function (LMF) whose parameters are denoted as $\left[a_{l}, b_{l}, c_{l}, d_{l}\right]$ where $c_{u}=d_{u}=c_{l}=d_{l}$. By using similarity of triangles, the values of $h$ and $n$ (marked in Fig. $1 \mathrm{~b}$ along the $\mu_{\tilde{L}}(x, u)$ axis) can be formulated as follows [11]:

$$
\begin{gathered}
h=\frac{b_{l}-b_{u}}{d_{u}-b_{u}}, h \in[0,1] \\
n=\frac{x^{\prime}-b_{u}}{d_{u}-b_{u}} \text { where } x^{\prime} \geq b_{u}, n \in[0,1]
\end{gathered}
$$

Note that $h$ is a constant once the parameters of the UMF and LMF are known whereas $n$ is dependent on $x^{\prime}$. Furthermore, the vertical slices of inputs $w, q$ and $s$ shown in Fig. $1 \mathrm{~b}$ are represented with a blue triangle having vertices $E^{w}, F^{w}, K^{w}$, a purple trapezoid having vertices $E^{q}, F^{q}, K^{q}, R^{q}$ and an orange singleton with height $R^{s}$,successively. In the mathematical formulation, the $3 \mathrm{D}$ coordinate system variables $\left(x, u, \mu_{\tilde{L}}(x, u)\right)$ are used where the points $E, F, K$ and $R$ in $3 \mathrm{D}$ space can be generalized letting $x=x^{\prime}$ as follows: $E=$ $\left(x^{\prime}, \underline{\mu_{\tilde{L}}}\left(x^{\prime}\right), 0\right), F=\left(x^{\prime}, \underline{\mu_{\tilde{L}}}\left(x^{\prime}\right), h\right), K=\left(x^{\prime}, \overline{\mu_{\tilde{L}}}\left(x^{\prime}\right), 0\right)$ and $R=\left(x^{\prime}, 1, \mu_{\tilde{L}}\left(x^{\prime}, 1\right)\right)$ (alternatively, $R=\left(x^{\prime}, 1, n\right)$ ) where $\mu_{\tilde{L}}\left(x^{\prime}\right)$ represents the lower membership degree of $x^{\prime}$ and $\overline{\mu_{\tilde{L}}}\left(x^{\prime}\right)$ represents the upper membership degree of $x^{\prime}$.

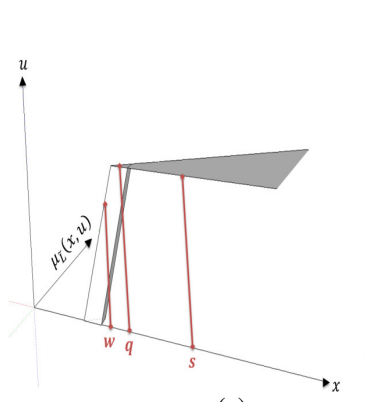

(a)

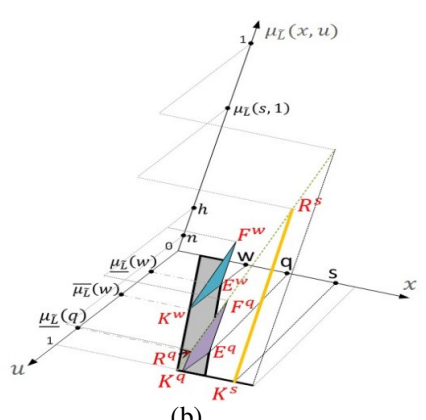

(b)
Fig. $13 \mathrm{D}$ view of an LGT2 FS showing a) the three singleton inputs $w, q$ and $s$ b) vertical slice representations: blue triangle is the vertical slice for input $w$, purple trapezoid is the vertical slice for input $q$, orange line is the vertical slice for input $s$

Now, the point value of $\mu_{\tilde{L}}\left(x^{\prime}, u\right)$ at $u=u^{\prime}$ can be written under three conditions depending on $x^{\prime}[11]$ :

1) Condition $1\left(x^{\prime} \leq b_{u}\right)($ see input $w$ in Fig. 1b):

$$
\mu_{\tilde{L}}\left(x^{\prime}, u^{\prime}\right)=\frac{h *\left(\overline{\mu_{\tilde{L}}}\left(x^{\prime}\right)-u^{\prime}\right)}{\left(\overline{\mu_{\tilde{L}}}\left(x^{\prime}\right)-\underline{\mu_{\tilde{L}}}\left(x^{\prime}\right)\right)}
$$

2) Condition $2\left(b_{u}<x^{\prime}<b_{l}\right)($ see input $q$ in Fig. $1 b)$ :

$$
=\frac{h *\left(\overline{\mu_{\tilde{L}}}\left(x^{\prime}\right)-u^{\prime}\right)-n *\left(\underline{\mu_{\tilde{L}}}\left(x^{\prime}\right)-u^{\prime}\right)}{\left(\overline{\mu_{\tilde{L}}}\left(x^{\prime}\right)-\underline{\mu_{\tilde{L}}}\left(x^{\prime}\right)\right)}
$$

3) Condition $3\left(x^{\prime} \geq b_{l}\right)$ : (see input $s$ in Fig. $\left.1 b\right)$ :

$$
\mu_{\tilde{L}}\left(x^{\prime}, u^{\prime}\right)=\frac{x^{\prime}-b_{u}}{d_{u}-b_{u}} \quad \text { where } x^{\prime} \geq b_{l} \text { and } u^{\prime}=1
$$

Note that Equation (7) does not depend on $u$ anymore due to the nature of the LGT2 FSs since the condition $x^{\prime} \geq b_{l}$ marks the shoulder part of the FS where $\mu_{\tilde{L}}\left(x^{\prime}\right)=\overline{\mu_{\tilde{L}}}\left(x^{\prime}\right)=1$. Also, it is important to note that $b_{l} \neq \overline{b_{u}}$ as the uncertainty bounds (FOU width) is predefined and conditions $b_{u}<b_{l}$ and $a_{u}<a_{l}$ hold for the creation of LGT2 FSs. Similarly, the parameters for the left shoulder LGT2 FS can be driven following the above procedure.

In order to represent General Type-2 (GT2) FSs, we have opted to use zSlices approach introduced by Wagner and Hagras [22]. However, the equivalence between alpha-plane and zSlices representations has been proven in [23]. Accordingly, LGT2 FSs can also be represented using alpha-planes introduced by Liu [24] and Mendel [25].

A zSlice $\tilde{Z}_{i}$ is formed by slicing a GT2 FS in the third dimension $(z)$ at level $z_{i}$ and is equivalent to an IT2 FS with the 
exception that its membership grade $\mu_{\tilde{Z}_{i}}(x, u)$ in the third dimension is not fixed to 1 ; instead is equal to $z_{i}$ where $0 \leq z_{i} \leq$ 1. Thus, the zSlice $\tilde{Z}_{i}$ can be written as follows [22]:

$$
\tilde{Z}_{i}=\int_{x \in X} \int_{u_{i} \in J_{i_{x}}} z_{i} /\left(x, u_{i}\right)
$$

where at each $x$ value, zSlicing creates an interval set with height $z_{i}$ and domain $J_{i_{x}}, \quad 1 \leq i \leq I$, and $I$ is the number of zSlices (excluding $\tilde{Z}_{0}$ ) and $z_{i}=i / I$.

\section{THE PROPOSED CONVERSION PARADIGM}

For comparison purposes, we have developed a conversion paradigm to redesign (using LGT2 FSs) the linguistic variables, which are modeled using EIA [10]. The proposed paradigm takes several key points within the parameters of IT2 fuzzy sets to redesign the linguistic variable using LGT2 FSs. The selection of the several key points depends on the number of the linguistic modifiers, hence the total number of linguistic terms. As a result, the number of linguistic terms modelled in the primary domain of an IT2 FS design is reduced to two (as a requirement of LGT2 FSs) and the modifiers (i.e. extremely, very, etc.) are modelled in the secondary domain. For example, let a linguistic variable named preparation time have the following linguistic terms: extremely short, very short, short, long, very long, extremely long. Using LGT2 FSs, the total number of primary membership functions (MFs) will be only two (having several zSlices), one MF for short and one MF for long; and the linguistic modifiers (i.e. extremely and very) will be modelled as secondary MFs (zSlices).

The developed conversion paradigm can be formalized as follows: Let $N$ be the total number of linguistic terms where $N>$ $3, N \in\{2 k: k \in \mathbb{Z}\}$; and $S$ be the total number of zSlices used in the representation of GT2 FSs. We define the following iteration variables (iter left $_{\text {and }}$ iter right $_{\text {) }}$ to be used in the paradigm where UMF is a trapezoidal function defined with parameters $\left[a_{u}, b_{u}, c_{u}, d_{u}\right]$ and LMF is a trapezoidal function defined with parameters $\left[a_{l}, b_{l}, c_{l}, d_{l}\right]$ :

$$
\begin{gathered}
\text { iter }_{\text {left }}=\left(d_{u_{\frac{N}{2}-2}}-c_{l_{0}}\right) /(S+1) \\
\text { iter }_{\text {right }}=\left(b_{l_{N-1}}-a_{u_{\frac{N}{2}+1}}\right) /(S+1)
\end{gathered}
$$

The indexes of the UMF and LMF parameters in Equations (9)-(10) indicate the linguistic terms that the parameters belong to (see Fig. 2). For example, $c_{l_{0}}$ is the third parameter of LMF $\left[a_{l}, b_{l}, c_{l}, d_{l}\right]$, which belongs to the first linguistic term having

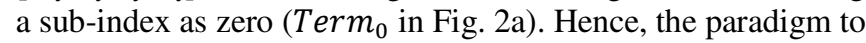
create LGT2 FSs using the parameters derived from EIA [10] can be formalized as below where the steps are categorized with regards to left shoulder MF and right shoulder MF:

\section{Converting IT2 FSs into LGT2 FSs}

\footnotetext{
Steps

0 Define the support of the linguistic variable: $\left[x_{\text {start }}, x_{\text {end }}\right]$

Left_1

Calculate iteration value iter $_{\text {left }}$ shown in Equation (9)
}

Left_2 Set currentpnt $=c_{l_{0}}$

For each zSlice $s$

Decide on the linguistic modifier by comparing currentpnt with linguistic modifier range (given by IT2 design)

Create $U M F_{S}$ and $L M F_{S}$ for zSlice $s$ using:

Left_4

$$
L M F_{S}=\left[x_{\text {start }}, x_{\text {start }}, \text { currentpnt }, b_{\frac{l_{N}-1}{2}}\right]
$$

$U M F_{s}=\left[x_{\text {start }}, x_{\text {start }}\right.$, currentpnt

$$
\left.+ \text { iter }_{\text {left }}, d_{u_{\frac{N}{2}-1}}\right]
$$

Right_1 Calculate iteration value iter $_{\text {right }}$ shown in Equation (10)

Right_2 Set currentpnt $=a_{u_{\frac{N}{2}+1}}$

For each zSlice $s$

Decide on the linguistic modifier by comparing

Right_3 currentpnt with linguistic modifier range (given by IT2 design)

Create $U M F_{S}$ and $L M F_{S}$ for zSlice $s$ using:

Right_4 UMF $F_{S}=\left[a_{\frac{u_{\frac{N}{2}}}{}}\right.$ currentpnt $\left., x_{\text {end }}, x_{\text {end }}\right]$

$$
L M F_{S}=\left[b_{l_{\frac{N}{2}}}, \text { currentpnt }+ \text { iter right }_{\text {rend }}, x_{\text {end }}\right]
$$

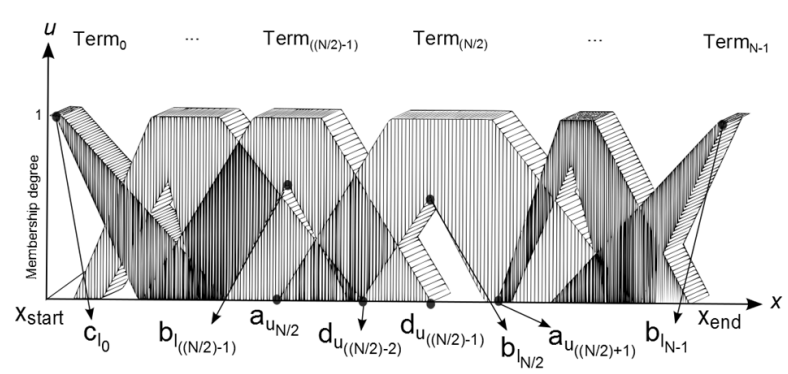

(a)

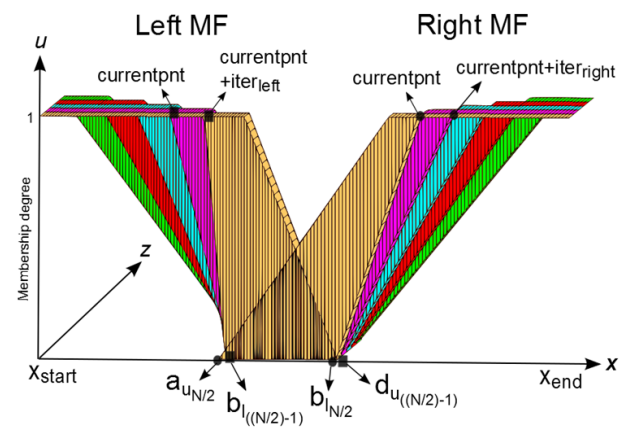

(b)

Fig. 2 The theoretical view of a) IT2 FS model b) LGT2 FS model showing the parameters and key points used in the conversion paradigm 


\section{COMPUTING WiTH WORDS FRAMEWORK}

The deployed CWWs architecture outputs LGT2 Fuzzy Sets [11], which can better represent the user perceptions while avoiding the drawbacks of type- 1 and interval type- 2 fuzzy sets and hence can establish a more natural human-machine interaction. The deployed CWWs framework can learn from user experiences and adapt to the events in order to link the computers and users in a humanlike manner. The architecture of the deployed CWWs Framework is briefly explained in the next subsection. Further details can be found in [16] [17].

\section{A. Architecture of the deployed Computing With Words Framework}

The deployed CWWs Framework has been blended from eclectic literature review about human problem solving behaviours from neuroscience, psychology, linguistics, cognitive science and artificial intelligence perspectives. The fundamental objective is to establish a natural communication by inputting natural language (words) and outputting natural language (words), which is a communication means to express ideas, feelings, thoughts, etc. used by the humans. Therefore, the validation of the generated word model can only be performed by the human, who will approve whether the output word matches his/her judgment. The validation can be assessed by obtaining feedback from the user.

In essence, Zadeh [26] stresses that there is a connection between the machinery of fuzzy logic and human reasoning. Furthermore, he groups the concepts underlying the human cognition into three: granulation, organization and causation [26]. These concepts are informally defined in [26] as follows: granulation involves decomposition of whole into parts; organization involves integration of parts into whole; and causation involves association of causes with effects. In view of Zadeh's definitions, the deployed CWWs Framework is divided into two segments, which are granulation and causationorganization as shown in Fig. 3.

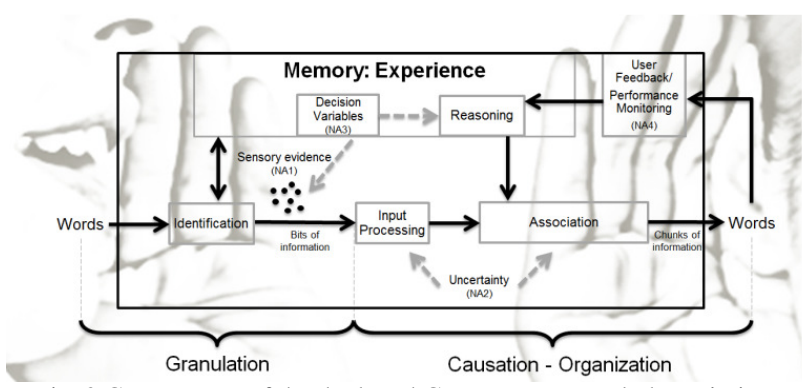

Fig. 3 Components of the deployed CWWs Framework that mimics humanlike communication (the black arrows show the direction of

information flow, and the grey dashed arrows show the possible impact factors that should be handled).

\section{B. Operation Principles of the deployed CWWs Framework}

The operation of the deployed framework is as follows: input words represent a problem that needs to be answered/solved and to do this; in granulation segment, the input words are first granulated by being mapped into sensory evidence of remembered solution in the human experience. The sensory evidence (bits of information) retrieved from the memory is regarded to be numerical descriptors of a solution that relates to the decision variables in human reasoning. For example, on an ordinary weekday, the user comes home from work tired and very hungry and s/he needs to prepare something very easy considering his/her status. The user's interpretation of 'very easy' depends on some criteria which happen to be the preparation time and the cooking time of the recipe. The problem descriptors in this case are tiredness and hungriness (in words), whereas the solution descriptors are preparation time and cooking time of the recipe in minutes (hence numerical). In other words, the identification element in the granulation segment takes tiredness and hungriness in words and outputs bits of information for preparation time and cooking time in numbers.

In causation-organization segment, the numerical sensory evidence is converted into words by input processing element so that the bits of information are classified to cope with the uncertainty associated to it in the human mind. The mapping of sensory evidence is done using fuzzy representations of the decision variables that characterize the human reasoning, which is represented in IF-THEN fuzzy rule format. For example, the decision variables in the previously mentioned scenario are preparation time and cooking time (linguistic variables), which have fuzzy representations using the linguistic labels 'short' vs. 'long' for the preparation time, and 'quick' vs. 'slow' for the cooking time. Moreover, the solution is described by the difficulty level of the recipe and has a fuzzy representation using the linguistic labels 'challenging' vs. 'easy'. So, in this scenario, the human reasoning is represented using fuzzy rules such as 'If preparation time is short and the cooking time is very quick then the difficulty level of the recipe is very easy'. Depending on the numerical inputs (bits of information), active rules are found by the association element and the output is drawn by first aggregating active rules into an interval format and then generalizing this interval into chunks of information (words) to be communicated back to the user. This concludes one way information flow of the causation-organization segment.

After the solution is presented to the user, for performance monitoring purposes, the output word needs to be evaluated by the user so that the deployed CWWs Framework can learn and adapt. This can be done by asking the user via natural language to provide interpretations for the decision variables and concludes the two way information flow in the causationorganization segment. For example, the user is asked to provide words for preparation time and cooking time as well as the difficulty level of the recipe in his/her opinion. Upon receiving this feedback, the human reasoning, which is in the form of IFTHEN fuzzy rules, can be modified to incorporate the incoming information, hence to learn. In summary, the deployed CWWs Framework follows a cyclic and integrated process of identifying in the granulation segment, and associating together with adapting in the causation-organization segment.

\section{EXPERIMENTS AND RESULTS}

We have conducted real world experiments with 17 subjects having different backgrounds. A survey composing of 18 words belonging to 3 linguistic variables has been distributed to collect interval data as suggested by EIA [10]. The participants were asked to indicate what the given words meant to them using an interval of $[0,10]$. The words to be modelled using EIA are as follows: for food preparation time linguistic variable: extremely short, very short, short, long, very long, extremely long; for 
cooking time linguistic variable: extremely quick, very quick, quick, slow, very slow, extremely slow, for overall time linguistic variable: (which is the summation of preparation time and cooking time) extremely little, very little, little, big, very big, extremely big.

As mentioned, the supports of the data-driven linguistic variable designs in both IT2 and LGT2 based CWWs Framework (i.e. $\left[x_{\text {start }}, x_{\text {end }}\right]=[0,10]$ ) were kept as $[0,10]$. Since the original domain of the linguistic variables might be different than $[0,10]$, the original domain of the input linguistic variables require to be scaled into the interval $[0,10]$. For example, for cooking time linguistic variable, the input can be 200 minutes whereas for preparation time linguistic variable, the maximum input value is 120 minutes. Hence, for a fair comparison, each linguistic variable should have its own minimum and maximum values in the mapping. However, this can cause disturbance if there are values that can be categorized as outliers. For example, for a recipe, we have recorded a cooking time of 400 minutes and that is quite far from the average of the all other recipes involved. Correspondingly, we have noticed in our experiments that changing the mapping of the linguistic variables causes greater disturbance in the RMSE and MAPE results belonging to IT2 based CWWs Framework than those belonging to LGT2 based CWWs Framework (see Section VI.B).

In this section, we will discuss three major comparison results between IT2 and LGT2 based CWWs Framework: first is the design of the word models, second is the statistical measures for error calculation, which are MAPE (Mean Absolute Percentage Error) and RMSE (Root Mean Square Error) based on the user feedback and the system responses together with the disturbance in these statistical measures caused by the different mappings of the original domain to the interval $[0,10]$, and third is the progressive MAPE and RMSE results for one participant due to space constraints.

Table I Parameters of 18 linguistic terms based on the survey data for IT2 based word models (using EIA)

\begin{tabular}{|c|c|c|c|c|c|c|c|c|c|}
\hline \multirow{2}{*}{ Words } & \multicolumn{4}{|c|}{ Upper Membership Function } & \multicolumn{5}{|c|}{ Lower Membership Function } \\
\hline & $a$ & b & c & d & $a$ & b & c & $d$ & u \\
\hline Extremely short & 0 & 0 & 0.183503 & 2.632993 & 0 & 0 & 0.091752 & 1.316497 & 1 \\
\hline Very short & 0.37868 & 1.5 & 2.5 & 4.62132 & 0.792893 & 1.75 & 1.75 & 2.207107 & 0.64644 \\
\hline Short & 1.37868 & 3 & 4 & 5.62132 & 2.585786 & 3.5 & 3.5 & 4.414214 & 0.64644 \\
\hline Long & 3.37868 & 5 & 6.5 & 8.62132 & 4.585786 & 5.6 & 5.6 & 6.414214 & 0.57573 \\
\hline Very long & 6.585786 & 7.5 & 0 & 9.414214 & 6.792893 & 7.666667 & 7.666667 & 8.207107 & 0.764298 \\
\hline Extremely long & 7.367007 & 9.816497 & 10 & 10 & 8.683503 & 9.908248 & 10 & 10 & 1 \\
\hline Extremely quick & 0 & 0 & 0.275255 & 3.94949 & 0 & 0 & 0.091752 & 1.316497 & 1 \\
\hline Very quick & 0.585786 & 1.5 & 2 & 3.414214 & 0.792893 & 1.666667 & 1.666667 & 2.207107 & 0.76429 \\
\hline Quick & 1.171573 & 3 & 4.5 & 6.828427 & 2.792893 & 3.6 & 3.6 & 4.207107 & 0.57573 \\
\hline Slow & 3.37868 & 5.5 & 6.5 & 7.62132 & 5.792893 & 6.25 & 6.25 & 7.207107 & 0.64644 \\
\hline Very slow & 5.37868 & 7 & 8 & 9.62132 & 6.792893 & 7.5 & 7.5 & 8.207107 & 0.64644 \\
\hline Extremely slow & 7.367007 & 9.816497 & 10 & 10 & 8.683503 & 9.908248 & 10 & 10 & 1 \\
\hline Extremely little & 0 & 0 & 0.183503 & 2.632993 & 0 & 0 & 0.091752 & 1.316497 & 1 \\
\hline Very little & 0.37868 & 2 & 3 & 4.62132 & 1.792893 & 2.5 & 2.5 & 3.207107 & 0.64644 \\
\hline Little & 1.37868 & 3 & 4 & 5.62132 & 2.585786 & 3.5 & 3.5 & 4.414214 & 0.64644 \\
\hline Big & 3.37868 & 5.5 & 7 & 8.62132 & 5.792893 & 6.4 & 6.4 & 7.207107 & 0.57573 \\
\hline Very big & 6.585786 & 7.5 & 8 & 9.414214 & 6.792893 & 7.666667 & 7.666667 & 8.207107 & 0.76429 \\
\hline Extremely big & 7.367007 & 9.816497 & 10 & 10 & 8.683503 & 9.908248 & 10 & 10 & 1 \\
\hline
\end{tabular}

A. Comparison of the design of the word models using IT2 and LGT2 fuzzy sets

As mentioned earlier, IT2 fuzzy sets have been derived from real-world user data for the inputs to the causation-organization segment of the deployed CWWs Framework. These inputs are preparation time, cooking time and overall time of the chosen recipe. In total, 18 words were investigated using the EIA [10] whose parameters are given in Table I. With this information, the whole design for one of the linguistic variables, for example preparation time, using IT2 fuzzy sets is illustrated in Fig. 4a.

For comparison purposes, the parameters of the LGT2 fuzzy sets including each zSlice derived using the paradigm described in Section IV are given in Table II.

The design of the LGT2 fuzzy set based models for one of the inputs (i.e. preparation time) to the causation-organization segment is given in Fig. 4b. The illustrated LGT2 fuzzy set has been created using the parameters in Table II and the paradigm described in Section IV. The linguistic modifiers marked as 'extremely', 'very' and 'none' are pointed with arrows in Fig. 4b and are modelled in the third dimension using zSlices representation.

Table II Parameters of each zSlice, hence linguistic term, derived using

\begin{tabular}{|c|c|c|c|c|c|c|c|c|c|}
\hline \multirow[b]{2}{*}{ Words } & \multirow{2}{*}{$\begin{array}{l}\text { Number } \\
\text { of zslice }\end{array}$} & \multicolumn{5}{|c|}{ the paradigm in Section IV } & \multicolumn{3}{|c|}{ Lower Membership Function } \\
\hline & & a & b & c & d & a & b & $\mathrm{c}$ & $\mathrm{d}$ \\
\hline $\begin{array}{l}\text { Extremely } \\
\text { short }\end{array}$ & 5 & 0 & 0 & 0.846679 & 5.62132 & 0 & 0 & 0.091751 & 3.5 \\
\hline \multirow{2}{*}{$\begin{array}{l}\text { very } \\
\text { short }\end{array}$} & 4 & 。 & 0 & 1.601608 & 5.62132 & 0 & 0 & 0.84668 & 3.5 \\
\hline & 3 & 0 & 0 & 2.356536 & 5.62132 & 0 & 0 & 1.601608 & 3.5 \\
\hline \multirow[b]{2}{*}{ Short } & 2 & 。 & 0 & 3.111464 & 5.62132 & 0 & 0 & 2.356536 & 3.5 \\
\hline & 1 & 。 & 0 & 3.866392 & 5.62132 & 0 & 0 & 3.111464 & 3.5 \\
\hline Long & 1 & 3.37868 & 6.585786 & 10 & 10 & 5.6 & 7.13953 & 10 & 10 \\
\hline \multirow[b]{2}{*}{ vervlong } & 2 & 3.37868 & 7.13953 & 10 & 10 & 5.6 & 7.693274 & 10 & 10 \\
\hline & 3 & 3.37868 & 7.693274 & 10 & 10 & 5.6 & 8.247017 & 10 & 10 \\
\hline \multirow{2}{*}{$\begin{array}{c}\text { Extremely } \\
\text { Iong }\end{array}$} & 4 & 3.37868 & 8.247017 & 10 & 10 & 5.6 & 8.800761 & 10 & 10 \\
\hline & 5 & 3.37868 & 8.800761 & 10 & 10 & 5.6 & 9.354505 & 10 & 10 \\
\hline \multirow{2}{*}{$\begin{array}{l}\text { Extremely } \\
\text { quick }\end{array}$} & 5 & 0 & 0 & 0.645495 & 6.828427 & 0 & 0 & 0.091752 & 3.6 \\
\hline & 4 & 0 & 0 & 1.199239 & 6.828427 & 0 & 0 & 0.645495 & 3.6 \\
\hline \multirow{2}{*}{$\begin{array}{l}\text { vern } \\
\text { quick }\end{array}$} & 3 & 0 & 0 & 1.752983 & 6.828427 & 0 & 0 & 1.199239 & 3.6 \\
\hline & 2 & 。 & 0 & 2.306726 & 6.828427 & 0 & 0 & 1.752983 & 3.6 \\
\hline Quick & 1 & 0 & 0 & 2.86047 & 6.828427 & 0 & 0 & 2.306726 & 3.6 \\
\hline \multirow{2}{*}{ Slow } & 1 & 3.37868 & 5.37868 & 10 & 10 & 6.25 & 6.133608 & 10 & 10 \\
\hline & 2 & 3.37868 & 6.133608 & 10 & 10 & 6.25 & 6.888536 & 10 & 10 \\
\hline \multirow{2}{*}{ very slow } & 3 & 3.37868 & 6.888536 & 10 & 10 & 6.25 & 7.643464 & 10 & 10 \\
\hline & 4 & 3.37868 & 7.643464 & 10 & 10 & 6.25 & 8.398392 & 10 & 10 \\
\hline $\begin{array}{l}\text { Extremely } \\
\text { slow }\end{array}$ & 5 & 3.37868 & 8.398392 & 10 & 10 & 6.25 & 9.15332 & 10 & 10 \\
\hline \multirow{3}{*}{$\begin{array}{c}\text { Extremely } \\
\text { litrle }\end{array}$} & 5 & 0 & 0 & 0.84668 & 5.62132 & 0 & 0 & 0.091752 & 3.5 \\
\hline & 4 & 。 & 。 & 1.601608 & 5.62132 & 0 & 0 & 0.84668 & 3.5 \\
\hline & 3 & 0 & 0 & 2.356536 & 5.62132 & 0 & 0 & 1.601608 & 3.5 \\
\hline \multirow{2}{*}{ very little } & 2 & 0 & 0 & 3.111464 & 5.62132 & 0 & 0 & 2.356536 & 3.5 \\
\hline & 1 & 0 & 0 & 3.866392 & 5.62132 & 0 & 0 & 3.111464 & 3.5 \\
\hline Big & 1 & 3.37868 & 6.585786 & 10 & 10 & 6.4 & 7.13953 & 10 & 10 \\
\hline \multirow{2}{*}{ Very big } & 2 & 3.37868 & 7.13953 & 10 & 10 & 6.4 & 7.693274 & 10 & 10 \\
\hline & 3 & 3.37868 & 7.693274 & 10 & 10 & 6.4 & 8.247017 & 10 & 10 \\
\hline \multirow{2}{*}{$\begin{array}{c}\text { Extremely } \\
\text { big }\end{array}$} & 4 & 3.37868 & 8.247017 & 10 & 10 & 6.4 & 8.800761 & 10 & 10 \\
\hline & 5 & 3.37868 & 8.800761 & 10 & 10 & 6.4 & 9.354505 & 10 & 10 \\
\hline
\end{tabular}

As illustrated below, among visual advantages of LGT2 FSs is their compact design, which is based on the use of antonyms. Employing LGT2 FSs reduces the number of MFs to be designed to two while keeping the same level of profoundness as in an IT2 design (see Fig. 4a). Hence, using LGT2 FSs not only simplifies the modeling process of a linguistic variable, but also decreases the number of fuzzy rules in the rulebase of a fuzzy logic system (FLS). Therefore, as previously shown in [16], [27], LGT2 FSs can facilitate the intelligent systems to respond faster. That is, the processing time of a complete rulebase (having all the combinations of the antecedents) of a LGT2 based FLS is significantly lower than the processing time of a complete rulebase of an IT2 based FLS. Furthermore, use of LGT2 FSs can diversify the output values of the system as LGT2 FSs are able to account for the small differences in the input. In other words, LGT2 FSs offer a richer (in terms of unique values) output range as they have a distinct secondary membership degree for every $x$ value in the universe of discourse, which in turn has a crucial impact on generating unique outputs. We have noticed that this impact can be better reflected when the LGT2 fuzzy sets are adapted with each 
incoming input. However, for this paper, both IT2 and LGT2 fuzzy sets have been fixed ahead of time based on the survey data collected from 17 subjects.

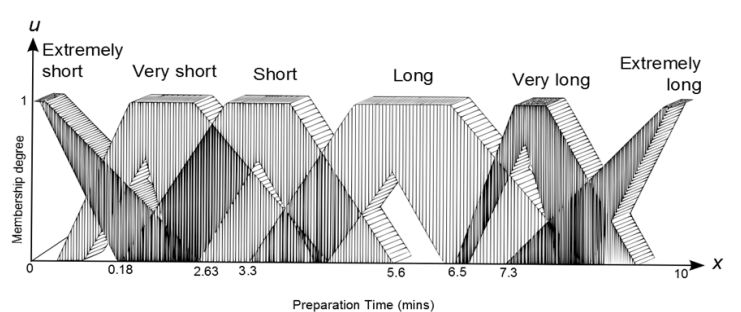

(a)

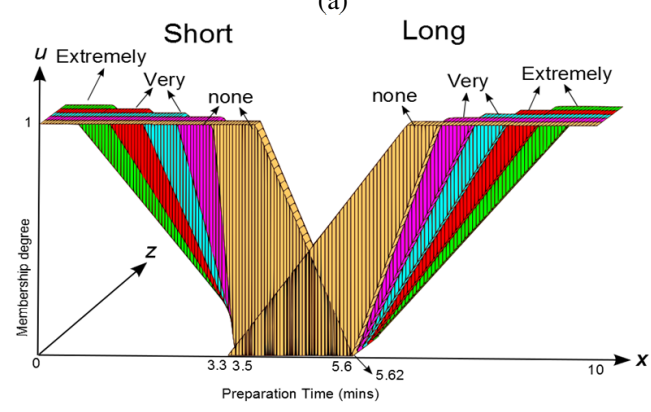

(b)

Fig. 4 (a) The whole IT2 word model for preparation time linguistic variable created using EIA scaled to the interval $[0,10]$ (b) The whole LGT2 word model for preparation time linguistic variable created using conversion paradigm and scaled to the interval $[0,10]$

\section{B. Comparison of the statistical measures RMSE and MAPE for IT2 and LGT2 based CWWs Framework}

In practice, the result of mimicking the human reasoning can be determined by comparing the classified outputs (words represented by numbers) using the distance (absolute value of the numerical difference between the system output and the user feedback). When the words are represented with numbers (extremely challenging: 6 , very challenging: 5 , challenging: 4 , easy: 3, very easy: 2 and extremely easy: 1), the absolute difference between the LGT2 based system response and the user feedback is significantly less when compared to the absolute difference between the IT2 based system response and the user feedback for all of the participants in the majority of the results.

There are two statistical measures employed for error calculation, which are MAPE and RMSE. In error calculation for this experiment, the abovementioned numerical representation was used for the linguistic terms. The formula used for the MAPE calculation is given in Equation (11) whereas the formula for RMSE calculation is given in Equation (12), where the variable $x S y s$ represents the system response in numbers and the variable $x$ User represents the user feedback in numbers.

$$
\begin{gathered}
\operatorname{MAPE}(x)=\frac{1}{n} \sum_{i=1}^{n}\left|\frac{x \text { Sys }-x U \text { ser }}{x \text { User }}\right| \\
\operatorname{RMSE}(x)=\sqrt{\frac{1}{n} * \sum_{i=1}^{n}(x \text { Sys }-x U \text { ser })^{2}}
\end{gathered}
$$

As mentioned earlier, we have noticed in our experiments that changing the minimum and maximum values in the input data that are used in the scaling of the linguistic variables causes greater disturbance in the RMSE and MAPE results belonging to IT2 based CWWs Framework than those belonging to LGT2 based CWWs Framework. In this section, we present the results of the experiments that change according to the minimum and maximum values [min, $\max$ ] for mapping of each linguistic variable to the interval $[0,10]$. Table III gives the corresponding values of mean and standard deviation (Std) of the MAPE and RMSE results (derived from 17 subjects) for both IT2 based and LGT2 based CWWs Framework as well as the improvement percentage for LGT2 based system over IT2 based system (calculated using: 100*(Mean of IT2 - Mean of LGT2) / Mean of IT2).

It can be observed from the results that the improvement of LGT2 based system can increase up to $55.43 \%$ for MAPE and to $36.77 \%$ for RMSE. Most importantly, we have noticed over four different scales that the disturbance caused by the change of the input domain is much more in an IT2 based system compared to LGT2 based system. For example, for another scale where preparation time domain is $[2,120]$, cooking time domain is $[2,420]$, and overall time domain is [5, 435], the mean of MAPE for LGT2 based system is $55.57 \%$ whereas the mean of MAPE for IT2 based system is $61.3 \%$. When compared to the mean values given in Table III, this can be interpreted as LGT2 based system can better handle the extreme value ranges in the input, and hence can be more robust when compared to IT2 based system for CWWs Framework.

Table III Results of the MAPE and RMSE calculations for LGT2 and IT2 based CWWs Framework where preparation time domain is [2, 120], cooking

\begin{tabular}{|c|c|c|c|c|}
\hline & \multicolumn{2}{|c|}{ MAPE } & \multicolumn{2}{|c|}{ RMSE } \\
\hline & LGT2 & IT2 & LGT2 & IT2 \\
\hline Mean & 48.984 & 109.904 & 1.596 & 2.525 \\
\hline Std & 16.835 & 32.080 & 0.340 & 0.371 \\
\hline $\begin{array}{c}\text { Improvement } \\
\text { of LGT2 over } \\
\text { IT2 }\end{array}$ & \multicolumn{2}{|c|}{$55.43 \%$} & \multicolumn{2}{|c|}{$36.77 \%$} \\
\hline
\end{tabular}
time domain is $[2,120]$, and overall time domain is $[5,150]$

\section{Comparison of the progressive MAPE and RMSE results for IT2 and LGT2 based CWWs Framework}

In our experiments, we have also recorded the progressive MAPE and RMSE, which are recalculated after each interaction, in other words, after each input. Herein, the results can be interpreted in terms of convergence regarding the decrease in the MAPE and RMSE. The faster the convergence, the better the learning and adaptation capabilities of the system. The progressive MAPE and RMSE results are illustrated in Fig. 5a and Fig. $5 b$ for one participant due to space constraints. The figures demonstrate the fast convergence for LGT2 based CWWs Framework as well as the lower overall MAPE and RMSE results compared to IT2 based CWWs Framework. Hence, it can be observed that LGT2 based CWWs Framework outperforms IT2 based CWWs Framework in the pace of learning and adaptation. 


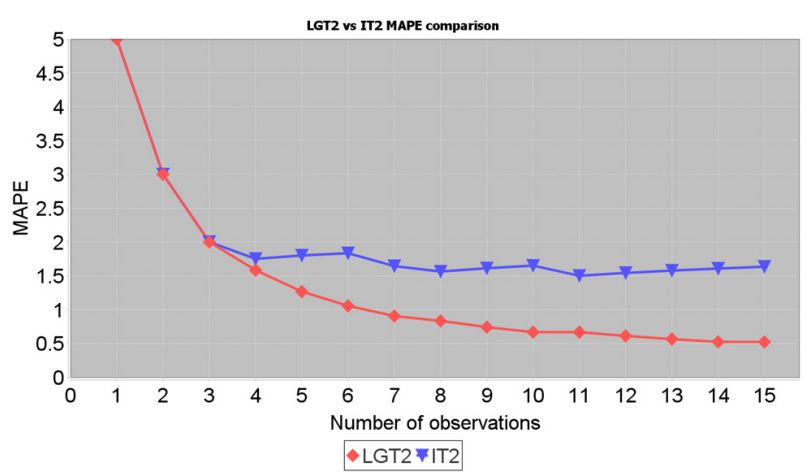

(a)

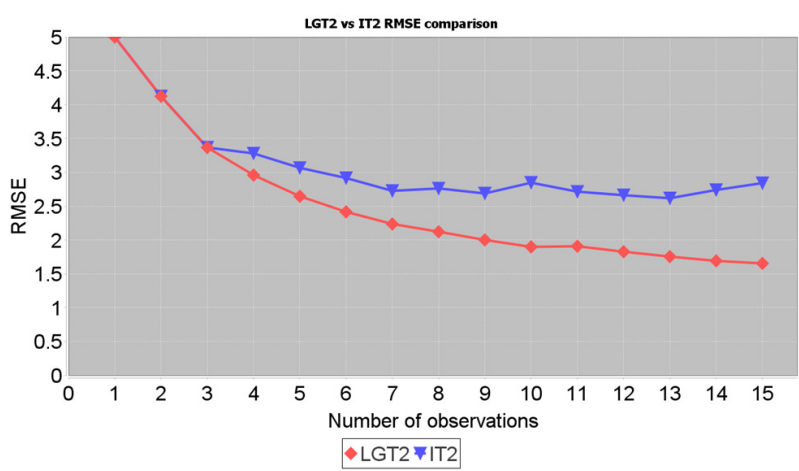

(b)

Fig. 5(a) Comparison of progressive MAPE for LGT2 and IT2 based CWWs Framework for Participant 7 (b) Comparison of progressive RMSE for LGT2 and IT based CWWs Framework for Participant 7

\section{CONCLUSIONS}

In this paper, we have presented a conversion paradigm that converts IT2 fuzzy sets, which are created using EIA, into LGT2 fuzzy sets. We have shown comparison analysis of IT2 and LGT2 based CWWs framework, where LGT2 based system's improvement reaches up to $55.43 \%$ for MAPE and to $36.77 \%$ for RMSE measurements. In compliance with the objective of CWWs paradigm, we showed that LGT2 fuzzy sets can be better capable of capturing and hence modelling perceptions for CWWs paradigm. Furthermore, LGT2 fuzzy sets were observed to outperform IT2 fuzzy sets regarding learning and adaptation capabilities as the progressive MAPE and RMSE values converge faster and are lower for LGT2 FSs.

\section{REFERENCES}

[1] L. A. Zadeh, "Outline of a New Approach to the Analysis of Complex Systems and Decision Processes," IEEE Transactions on Systems, Man, And Cybernetics, vol. SMC-3, no. 1, pp. 28-44, 1973.

[2] L. Zadeh, "Fuzzy logic = computing with words," IEEE Transactions on Fuzzy Systems, vol. 4, no. 2, pp. 103-111, 1996.

[3] L. Zadeh, "From Computing with Numbers to Computing With Words From Manipulation of Measurements to Manipulation of Perceptions," Int. J. Appl. Math. Comput. Sci, vol. 12, no. 3, pp. 307-324, 2002.

[4] J. Mendel, "Computing with words, when words can mean different things to different people," in Proceedings of the 3rd International ICSC
Symposium on Fuzzy Logic and Applications, Rochester, NY, pp. 158164, June 1999.

[5] J. Mendel, "Type-2 fuzzy sets: some questions and answers," IEEE Connections, vol. 1, pp. 10-13, 2003.

[6] J. Mendel, "An architecture for making judgments using computing with words," International Journal of Applied Mathematics and Computer Science, vol. 12, no. 3, pp. 325-336, 2002.

[7] J. Mendel, and $\mathrm{D}$. Wu, "Perceptual Reasoning for Perceptual Computing," IEEE Transactions on Fuzzy Systems, vol. 16, no. 6, pp. 1550-1564, 2008.

[8] J. Mendel, and D. Wu, Perceptual computing: Aiding people in making subjective judgments. Hoboken, NJ, USA: John Wiley \& Sons, 2010.

[9] F. Liu and J. M. Mendel, "Encoding words into interval type-2 fuzzy sets using an Interval Approach," IEEE Trans. Fuzzy Syst., vol. 16, no. 6, pp. 1503-1521, Dec. 2008

[10] D. Wu, J. M. Mendel, S. Coupland, "Enhanced Interval Approach for Encoding Words Into Interval Type-2 Fuzzy Sets and Its Convergence Analysis," IEEE Transactions on Fuzzy Systems, vol.20, no.3, pp. 499513, June 2012.

[11] A. Bilgin, H. Hagras, A. Malibari, M. Alhaddad and D. Alghazzawi, "Towards a general type-2 fuzzy logic approach for Computing With Words using linear adjectives," in 2012 IEEE Int. Conference on Fuzzy Systems, Brisbane, 2012.

[12] S. Greenfield, and R. John, "The Uncertainty Associated with a Type-2 Fuzzy Set," Views on Fuzzy Sets and Systems from Different Perspectives Philosophy and Logic, Criticisms and Applications, Studies in Fuzziness and Soft Computing, vol. 243, Springer-Verlag, pp. 471-483, 2009.

[13] E. Klein, "A semantics for positive and comparative adjectives," Linguistics and Philosophy, vol. 4, no. 1, pp. 1-45, 1980.

[14] E. Trillas, and S. Guadarrama, "What about fuzzy logic's linguistic soundness?," Fuzzy Sets and Systems, vol.156, no.3, pp. 334-340, 2005.

[15] C. Kennedy, Projecting the adjective: The syntax and semantics of gradability and comparison. Routledge, 1999.

[16] A. Bilgin, H. Hagras, A. Malibari, M. Alhaddad and D. Alghazzawi, "An experience based linear general type-2 fuzzy logic approach for Computing With Words," in 2013 IEEE Int. Conference on Fuzzy Systems, Hyderabad, 2013.

[17] A. Bilgin, H. Hagras, A. Malibari, M. Alhaddad and D. Alghazzawi, "A computing with words framework for ambient intelligence," in 2013 IEEE Int. Conference on Sys., Man and Cybernetics, Manchester, 2013.

[18] C. Kennedy, L. McNally, "Scale structure, degree modification, and the semantics of gradable predicates." Language (2005): 345-381.

[19] R. Schwarzschild, "Measure phrases as modifiers of adjectives." Rechercheslinguistiques de Vincennes 34 (2005): 207-228.

[20] C. Kennedy, "Polar opposition and the ontology of 'degrees'." Linguistics and philosophy, vol. 24, no. 1 (2001): 33-70.

[21] J. Mendel, Uncertain Rule-Based Fuzzy Logic Systems: Introduction and New Directions, Upper Saddle River, NJ: Prentice-Hall, 2001.

[22] C. Wagner, H. Hagras, "Toward general type-2 fuzzy logic systems," IEEE Trans. on Fuzzy Systems, vol. 18, no. 4, pp. 637-660, 2010.

[23] D. Zhai, and J. M. Mendel, "Comment on "Toward General Type-2 Fuzzy Logic Systems Based on zSlices"”, IEEE Transactions on Fuzzy Systems, 20(5), 996, 2012.

[24] F. Liu, “An efficient centroid type-reduction strategy for general type-2 fuzzy logic system,” Information Sciences, 178, 9, pp. 2224-2236, 2008.

[25] J. M.Mendel, L. Feilong, and Z. Daoyuan, “-Plane representation for type2 fuzzy sets: theory and applications.” Fuzzy Systems, IEEE Transactions on 17, no. 5 (2009): 1189-1207.

[26] L. Zadeh, "Toward a theory of fuzzy information granulation and its centrality in human reasoning and fuzzy logic," Fuzzy Sets and Systems, vol. 90, no. 2, pp. 111-127, 1997.

[27] A. Bilgin, H. Hagras, A. Malibari, M.J. Alhaddad and D. Alghazzawi, "Towards a linear general type-2 fuzzy logic based approach for computing with words," Soft Computing, pp. 1-20, 2013. 